\title{
Cuerpos y mentes para el trabajo: la psicologización de los trabajadores en Bogotá y Medellín, 1946-1991
}

\author{
Bodies and minds for \\ work: the psychologization \\ of workers in Bogotá and \\ Medellín, 1946-1991
}

CASTAÑO GONZÁLEZ, Eugenio. Cuerpos y mentes para el trabajo: la psicologización de los trabajadores en Bogotá y Medellín, 1946-1991. História, Ciências, Saúde - Manguinhos, Rio de Janeiro, v.27, n.2, abr.-jun. 2020, p.447465.

\section{Resumen}

En este artículo se pretende indagar la emergencia y desarrollo de una serie de discursos psicológico-administrativos sobre la motivación laboral, el clima organizacional y la calidad de vida en el trabajo, en ciudades como Bogotá y Medellín. Con ello se trata de dar cuenta del auge y expansión del conocimiento psicológico aplicado al mundo del trabajo y orientado a la optimización de rendimientos. La puesta en marca de estrategias de medición y cuantificación se erigieron en herramientas destinadas a objetivar comportamientos y fomentar una especie de reingeniería socio-laboral, en medio de un contexto caracterizado por la creciente inestabilidad laboral y el incremento del desempleo a finales de la década de 1980 y comienzos del 1990.

Palabras clave: fuerza laboral; recurso humano; motivación; clima organizacional.

\section{Abstract}

This article seeks to explore the emergence and development of a series of psychological and managerial discourses on worker motivation, organizational climate and quality of life at work in cities like Bogotá and Medellin. This sheds light on the rise and expansion of psychological knowledge applied to the world of work, aimed at optimizing productivity. Strategies for measurement and quantification were put in place that became tools for objectifying behaviors and encouraging a sort of social and labor re-engineering, within the context of growing labor instability and rising unemployment in the late 1980s and early 1990 s.

Keywords: workforce; human resource; motivation; organizational climate. 
$\mathrm{E}^{n}$ la primera parte de este artículo se examinará la manera en que la motivación psicológica se constituyó en una estrategia orientada a fomentar actitudes proclives al trabajo productivo. También se analizará la forma en que se diseñaron estrategias de medición y cuantificación de la satisfacción y motivación laboral. En la segunda parte, y articulándose con los desarrollos previos sobre la motivación, se examinará la emergencia, alcances, estrategias y objetivos de medición de lo que se definió como clima organizacional y la calidad de vida en el trabajo a partir de la década de 1970 y 1990, respectivamente. Lo anterior buscó hacer de la personalidad un recurso económico y psicológico provechosos para las empresas, la productividad, la satisfacción y la presunta felicidad de los trabajadores, en un contexto de creciente incertidumbre e inestabilidad laboral (Laval, Dardot, 2013, p.364). En síntesis, con este artículo se procura dar cuenta de un progresivo proceso de psicologización del mundo del trabajo a partir de la segunda mitad del siglo XX, en plena etapa de transformación del aparato productivo.

En términos metodológicos, los tipos de fuentes consultadas y examinadas fueron: libros, tesis de grado, artículos de revista científica y especializada, artículos de revista y de prensa no especializada, además de magazines de divulgación, como Fabricato al Día. Con la elección de este tipo de fuentes se procuró dar cuenta de tres asuntos claves a la hora de problematizar históricamente estos dispositivos psicológicos. En primer lugar, se decidió examinar el modo en que circularon, apropiaron y difundieron aquellos discursos y prácticas, incluso, más allá de las fronteras estrictamente disciplinares de la psicología, como la administración y la ingeniería. En segundo lugar, se decidió indagar varios tipos de publicaciones: unas con un contenido científico-académico y otras con una pretensión más divulgativa. En tercer lugar, la elección metodológica de los autores de las fuentes consultadas no respondió exclusivamente a la necesidad de referenciarlos como médicos, psicólogos, administradores, entre otros, sino más por el modo de problematizar la circulación de estos dispositivos "psi" dentro del ámbito laboral. En síntesis, la recolección y análisis cualitativo de estas fuentes respondió a la necesidad de problematizar dichos fenómenos en medio de un contexto histórico caracterizado por las transformaciones del aparato productivo.

La periodización elegida responde a dos fenómenos claves y vigentes: en primer lugar, la publicación de la Introducción al estudio del factor humano en la industria: nociones de psicoeconomía (Madariaga, 1946). Así, a partir de esta publicación se fueron consolidando los estudios sobre el factor humano, la motivación y cooperación en el ámbito laboral colombiano, tomando como punto de referencia los saberes psicológico-administrativos. La fecha de cierre corresponde al artículo "Calidad de vida en el trabajo: alcances e implicaciones", escrito por Fernando Toro Álvarez en 1991. A partir de esta fecha, a juzgar por las fuentes disponibles, los planteamientos sobre el clima organizacional comenzaron a instaurarse como parte de una estrategia de racionalización psicológica en los ámbitos laborales en ambas ciudades.

Por otro lado, la elección de las ciudades de Bogotá y Medellín para el rastreo de fuentes obedeció a tres motivos: el primero, porque ambas ciudades, desde finales del siglo XIX y comienzos del XX se constituyeron en los principales centros urbanos del país. En segundo lugar, porque ambas se establecieron como los principales núcleos del proceso de industrialización durante todo el siglo XX en Colombia. No obstante, a pesar de que el 
proceso de industrialización a nivel nacional tuvo otros polos de desarrollo (Ocampo, 1987), es importante aclarar que el enfoque metodológico no se orienta a analizar, en términos históricos, dicho proceso. Sin embargo, sí se procura identificar, a grandes rasgos y evitando incurrir en posiciones deterministas, cómo ciertas transformaciones socioeconómicas coincidieron con las propias modificaciones en los dispositivos psicológicos dentro del ámbito laboral. En tercer lugar, y partiendo de los dos motivos anteriores, así como de las fuentes disponibles para el desarrollo del presente artículo, estas dos ciudades se erigieron en los primeros y principales centros de divulgación de saberes sobre los procesos de psicologización de los trabajadores colombianos, lo que no significa que fuesen los únicos. Las fuentes consultadas en ambas ciudades dan cuenta de un tipo de discurso y de práctica que procura tener implicaciones nacionales. No obstante, a raíz de la periodización existen fuentes que solo pudieron ser examinadas de manera parcial por la vigencia de la ley n.23 de 1982 sobre derechos de autor, la cual limitó la consulta y reproducción de una parte de dichas fuentes, especialmente las tesis. Su disparidad, heterogeneidad y escasa disponibilidad tornó difícil examinar ambas ciudades como estructuras analíticas separadas dentro del artículo; aun cuando, en la medida de lo posible, se evitó establecer generalizaciones con alcances nacionales en las interpretaciones realizadas. Por ello la decisión metodológica de elaborar una distribución narrativa basada en el escrutinio detallado de las características y modificaciones apreciadas en los discursos y prácticas psicológicas en el ámbito laboral. De igual modo fue importante articular lo anterior con algunos cambios socioeconómicos experimentados durante el periodo propuesto con el propósito de realizar un análisis histórico más profundo.

Al revisar la producción historiográfica a nivel internacional se encuentran algunos enfoques de trabajo. El primero de ellos, desarrollado por autores como Sarraceno (2013), Billiard (2002), Ouvrier-Bonnaz (2010), Le Bianic (2004) y Vatin (2004), se centra en el análisis de la sociedad salarial europea del siglo XX. Este enfoque examina cómo se desarrolló el estudio de la actividad corporal y mental de los trabajadores desde una perspectiva psicofisiológica. Para ello abordan el asunto de la orientación, selección y reclutamiento de obreros durante la primera mitad del siglo XX, con el objetivo de determinar las aptitudes laborales desde la psicología experimental. Si este primer enfoque vuelca la atención en la primera mitad del siglo XX, los estudios sobre la gubernamentalidad desarrollados en el ámbito anglosajón, en cabeza de Nikolas Rose (1990), se ocupan de la implementación de prácticas de subjetivación laboral en el ámbito europeo y norteamericano durante los siglos XX y XXI, partiendo de ciertos discursos psicológico-administrativos. En el ámbito latinoamericano cobran un valor de capital importancia los trabajos de autores como Gallo Vélez (2016b), Caponi (2015), Haidar (2016), Roldan (2010), Motta (2008) Pulido Martinez (2008) y Jaraba Barrios (2014). Con base en las pesquisas desarrolladas por estos autores es posible identificar cuatro ámbitos de trabajo muy vinculados. El primero corresponde a la exploración de fenómenos como la fatiga en el ámbito laboral y sus formas de objetivación durante la primera mitad del siglo XX. Se trata de un tipo de análisis histórico que pone de relieve - en un contexto de creciente industrialización - la intención de regular los cuerpos para reformar las mentes con el fin de optimizar rendimientos. Un segundo ámbito de trabajo se desprende de las pesquisas históricas sobre el concepto del factor humano - 
vinculado con la emergencia de la fatiga y otros fenómenos como la psicotecnia - el cual procuró dar un paso adelante en ese proyecto de psicologización del ámbito laboral y de los análisis del cuerpo humano desde la metáfora del "motor". El tercero se enfoca en el problema de la simulación. Se trata de resaltar el rol desempeñado por la psiquiatría francesa, la práctica médico-legal y la medicina del trabajo en la objetivación de este padecimiento en situaciones de accidentalidad laboral (Gallo Vélez, 2016b). Existe un punto en común entre estos tres ejes investigativos en torno al esclarecimiento de los mecanismos orientados a la optimización de los rendimientos laborales desde el plano emocional, guiados por una experiencia de reingeniería permanente del cuerpo y la mente en las sociedades modernas y contemporáneas. Aunque no corresponden estrictamente a investigaciones de carácter historiográfico, también es posible señalar un cuarto ámbito investigativo desarrollado por Hernán Camilo Pulido Martínez sobre la psicología y el mundo laboral. Sus valiosos aportes se centran en exploraciones sobre la psicologización del servicio urbano de trabajadores en Bogotá (Pulido Martínez, 2008) y en las reflexiones teórico-conceptuales sobre los enfoques psicológicos ligados al ámbito organizacional. Estos trabajos de Pulido son bien interesantes en la medida que revelan una práctica que se ha venido extendiendo en los ámbitos laborales colombianos durante los últimos años.

Finalmente, existe un quinto ámbito de trabajo realizado por Bruno Jaraba sobre la fundación de la psicología en Colombia y de las herramientas psicotécnicas. Si bien su aporte es valioso al brindar pistas sobre la implementación de estas herramientas por la española Mercedes Rodrigo en la Universidad Nacional, su análisis se restringe a los procesos de admisión a dicho centro educativo y no aborda las implicaciones de estos dispositivos psicológicos en los ámbitos laborales. Por ello con este artículo se pretende examinar un fenómeno bastante vigente en las discusiones ligadas con la psicología organizacional, pero poco explorado por la historiografía nacional como es el tema de la psicologización del mundo del trabajo en Colombia y a partir de los siguientes aspectos: la motivación, la satisfacción, el clima organizacional y la calidad de vida en el mundo del trabajo durante la segunda mitad del siglo XX.

\section{De la metáfora del motor a la estrategia de la psicologización}

A finales del siglo XIX y comienzos del XX la metáfora de la sociedad y los individuos como máquinas térmicas se erigió en una rica fuente de análisis sobre la productividad y la necesidad de evitar el desgaste del "motor humano" (Pohl-Valero, 2010, p.42). La maximización del cuerpo como motor se orientó hacia el ahorro de energía y la optimización en el movimiento de los músculos y los nervios. Lo anterior también hizo parte de una intención por conquistar los efectos negativos de la mala organización, la explotación y las labores irregulares (Rabinbach, 1990, p.10). No obstante, la creciente humanización laboral generó la necesidad de incorporar elementos motivacionales, compensaciones y reconocimientos no sujetos a medidas de tipo pecuniario, con lo cual la metáfora del "motor" comenzó a evidenciar cierto desgaste. Se trató de un modo de internalizar la voluntad de trabajo y despertar una conducta proclive a la felicidad del individuo (Friedman, 1971, p.117). El centro de atención se orientó, desde la motivación por mantener la 
productividad, a la motivación y satisfacción del individuo en el lugar de trabajo (Aquilino, 2004). El entrecruzamiento entre las emociones y el ámbito de acción económica impulsó un discurso psicológico y administrativo que buscó atender la dimensión presuntamente irracional del trabajo para aumentar las ganancias. Además procuró combatir los conflictos laborales, neutralizar la lucha de clase (Illuz, 2006, p.46) y evitar al máximo los estragos de la inadaptación, vistos como factores desencadenados por el carácter desgastado de la psique (Sennett, 2000). En síntesis, la economía de los movimientos, el ajuste a las condiciones físicas, los problemas de monotonía, entre otros, fueron cimentando la base de un enfoque destinado a interrogar la psicología del trabajador. Ello tuvo como consecuencia la centralización de la atención hacia los problemas de la motivación, las interacciones sociales, afectivas y al análisis de las estrategias de los actores (Dejours, 1998, p.14). Los problemas económicos, la insatisfacción con diferentes aspectos de la vida y del trabajo, los conflictos morales, los problemas familiares, la felicidad, entre otros, se constituyeron en nuevos focos de atención dentro del proceso productivo (Gallo Vélez, 2016a, p.244). De acuerdo con lo afirmado por Dejours (1998, p.15), la visión del trabajador, visto como recurso, se ajustaba a una pretensión orientada a su motivación a partir de lo desarrollado por las ciencias de la administración y la psicología industrial.

Según Gallo Vélez (2016a), durante las dos primeras décadas del siglo XX en la Escuela de Minas y la Empresa Minera el Zancudo, se realizaron algunos desarrollos incipientes en torno a la atención médico social y la motivación laboral por intermedio de Juan de la Cruz Posada, Antonio José Álvarez, Alfonso Mejía y Alejandro López. Uno de los aspectos más notables de los planteamientos desarrollados por este último fue el de la economía industrial. De allí se fue desplegando el anhelo de investigar al individuo en su mentalidad, aptitudes, motivación y sentimientos experimentados en el lugar de trabajo (López, 2011, p.36).

\section{Motivar para trabajar}

Fue con el ingeniero español César de Madariaga que las reflexiones sobre este aspecto experimentaron un giro notorio. Su arribo al país durante la década del 1940 se dio como efecto de los procesos migratorios desencadenado por la Guerra Civil Española y la posterior dictadura de Francisco Franco (1939-1975). Formado en ingeniería de minas y metalurgia, era un conocido promotor y defensor de los beneficios de la organización científica del trabajo. También se desempeñó en Colombia como profesor de la Facultad de Administración Industrial y Comercial (Faic) del Gimnasio Moderno, precursora de la Facultad de Economía de la Universidad de Los Andes. Para él era esencial volcar la atención hacia los problemas de orientación, motivación, características psicológicas individuales y colectivas de cada grupo geoeconómico. Por aquel entonces el país transitaba por un proceso de transformación del aparato productivo y de su estructura laboral. La sustitución de importaciones de bienes livianos, intermedios y duraderos, permite comprender cómo esta estrategia de racionalización psicológica coincidió con un contexto socioeconómico muy diferente al de comienzos de siglo. La intervención estatal, producto de las restricciones del flujo comercial ocasionado por la Segunda Guerra Mundial, se hizo mucho más notoria en temas como el control de importaciones y exportaciones, otorgamiento de 
subsidios directos e indirectos a empresas industriales, participación del sector público en la relación entre proveedores, productores y canales de distribución. A lo anterior se le sumó la creación del Instituto de Fomento Industrial en 1940 por el entonces presidente Eduardo Santos, lo cual favoreció el montaje de la industria química, caucho y metalúrgica (Garay, 1998). A ello se le añadió que la segunda pos guerra dio paso a la consolidación de otras actividades económicas como el transporte, sector financiero, comunicaciones, servicios públicos modernos. Lo anterior se tradujo en el incremento del trabajo asalariado (distribuido en peones, obreros y empleados) entre finales de la década de 1930 y comienzos de la de 1960 (Sánchez, 2009, p.173). En efecto, según Ocampo (1987), el sector servicios acrecentó su participación en la generación de ocupaciones del 21\% en 1938 al 45\% en 1984. Así, el incremento de los dispositivos fabriles y del trabajo asalariado puso en juego una creciente atención a la motivación psicológica en las relaciones laborales como fuente de productividad y gestión de la subjetividad.

La coincidencia temporal entre estos cambios socioeconómicos y la emergencia de los discursos psicológicos sobre la motivación laboral, permite plantear el siguiente interrogante: ¿De qué manera se fue desplegando el interés por motivar psicológicamente a los trabajadores? Para responder de manera tentativa a este interrogante es fundamental plantear el análisis alrededor de dos aspectos claves: la cooperación y motivación como estrategia de estímulo emocional, con la pretensión de incrementar la productividad. Lo anterior invita a un rápido examen comparativo entre lo planteado por Alejandro López y César de Madariaga. Para el primero era claro que todo tipo de trabajo implicaba un ejercicio de cooperación necesario e inevitable, en tanto el trabajo de uno suplía las deficiencias del otro. Los métodos ideados para obtener la colaboración debían poner en juego un conjunto de fuerzas anímicas en la consecución de un objetivo común (López, 2011, p.95). Por otro lado, en Madariaga la cooperación y motivación no solo redundaba en provecho de la empresa, sino que, por un lado, su puesta en marcha dependía de una serie de factores psicológicos en empresarios y trabajadores; $y$, por otro lado, también radicaba en la posibilidad de desplegar la capacidad conjunta de esfuerzos, mentalidades y voluntades. Al mismo tiempo, no solo examinó el papel de la cooperación en los lugares de trabajo, sino que estableció una serie de tipologías alrededor de las políticas de cooperación y motivación en las empresas (Madariaga, 1946, p.115). Si bien en López y en Madariaga, aspectos como la motivación estuvieron presentes en sus planteamientos, durante la segunda mitad del siglo XX fue mucho más evidente y detallada esta concepción del individuo. Lo anterior partía de la idea de concebirlo como un actor sobre el cual debía de suscitarse un conjunto de conductas motivacionales, tomando en consideración, por un lado, las características emotivas y personales que debía poseer el líder empresarial, y, por otro lado, las condiciones emocionales que debían promoverse en el obrero y empleado en plena etapa de sustitución de importaciones.

La promoción de nuevas ideas sobre motivación, liderazgo, participación y trabajo en grupo, se articuló con la creación de departamentos de dinámica organizacional en empresas como Bavaria, Telecom, Sena, Colseguros, Intercol, entre otras (Dávila, 2001, p.219). Así mismo, en octubre de 1964, Jaramillo (1964, p.29) publicó en la revista Fabricato al Día un artículo titulado "Adaptación de la empresa al hombre". Para él, las políticas de relaciones 
humanas suponían la buena adaptación del individuo a su grupo de trabajo y a la tarea que debía ejecutar en cualquier empresa colombiana. Por dicha razón se debía analizar los diferentes grupos de trabajo y estar al tanto de las relaciones globales en cada uno, con el objetivo de tener un mejor contacto entre la dirección y los obreros. En consecuencia, el desarrollo organizacional, el interés y la motivación se constituyeron, en empresas como Fabricato, en una herramienta que tuvo como finalidad producir con calidad y a bajo costo (Lotero Zapata, 1964, p.19). La reivindicación del obrero como "actor", en empresas como esta, se instauró en un recurso valioso para buscar estimular actitudes propositivas en la masa obrera (Orientación, 1960, p.11).

Por otro lado, la rutinización y simplificación de las técnicas de trabajo, la fragmentación y mecanización de los procesos de producción, requirieron de un análisis sobre la pérdida del interés del trabajador. De allí la necesidad de reacondicionar psicológicamente al individuo en los lugares de trabajo, posibilitando con ello la recuperación del interés, el mejoramiento del ambiente laboral y los planes de ascenso. A comienzos de la década de 1960 también se fue haciendo perentorio explorar cómo el principio de la motivación debía articular los objetivos personales con los organizacionales. Durante ese mismo periodo y, desde la Escuela de Administración Pública (Esap), ubicada en la ciudad de Bogotá, comenzó a irrumpir un incipiente imperativo de la competencia como forma de estimular la motivación en los trabajadores desde varios ángulos: motivación por medio de la participación; por medio de los objetivos, tanto personales como organizacionales; por la competencia; por el desafío; por el cultivo de la atención; por el cambio de actitud (Ramírez Cardona, 1963, p.113). La metáfora deportiva se constituyó en un elemento clave para proclamar directamente las bondades de la productividad, el entusiasmo y el desafío, a través de un mayor sistema de puntuación (p.68).

Lo anterior se tradujo en el propósito de articular la fuerza de la motivación y el liderazgo para que la administración desarrollara en los individuos una serie de competencias, capaces de ser incorporadas como normas de vida. La reducción de los márgenes de ganancia, como los reportados en Fabricato en el mes de agosto de 1964, fue una razón suficiente para buscar alternativas que contribuyeran a optimizar la "atmosfera psicológica". En general, esta atmosfera pregonada en algunas publicaciones de Fabricato al Día, durante la primera mitad de la década de 1960 (Satizabal, ago. 1964, p.26), hizo primar a los individuos y su iniciativa por sobre la maquinaria y las instalaciones físicas. La observación detallada y metódica, esta especie de inmersión en los diversos espacios de trabajo se fue constituyendo, según demandaban autores como Satizábal (p.26), en un factor determinante que debía poseer y potencializar un supervisor en empresas para reforzar las condiciones positivas en la ejecución de una labor. A su vez, la eficiencia, la rapidez, educación, atención, satisfacción, también se volvieron cualidades que debían contribuir a mejorar aquella "atmosfera psicológica" y la motivación (Montoya, sep.-oct. 1966, p.8).

Dicha motivación debía estar fundamentada en el desarrollo de cierto nivel de docilidad en el ámbito laboral. Se trataba con ello de promover la capacidad de alternar varias actividades, dedicarse a la vida familiar, cordial, afectuosa, agradable, manteniéndose activo, enérgico, "diciéndole si a todo", evitando subestimarse y evadirse de los problemas emocionales (Montoya, sep.-oct. 1966, p.9). Promover actitudes positivas, cordiales, 
amistosas con los compañeros, nutría "el mágico pulmón de la salud mental" (p.9). Dicho en otros términos, este enfoque motivacional anhelaba promover comportamientos dóciles, estabilizados emocionalmente, neutralizadores de la "lucha de clases" (Illuz, 2006, p.46), proclives a evitar todo tipo de sobresaltos que transgrediese, no solo la maquinaria mental, sino también la jerarquía organizacional y social en pleno auge.

Pero fue en el Departamento de Ingeniería de la Universidad de los Andes donde, a partir de 1971, adquirió mayor peso el tema de la motivación en las organizaciones por medio de la oferta de un pensum académico específico que versaba sobre los siguientes asuntos: Aprendizaje y motivación social, Dinámica de la organización I y II y Seminarios de desarrollo organizacional. La preocupación por estos nuevos temas vinculados con la maximización de las capacidades laborales, en un espacio académico donde acudía gran parte de la élite política y económica colombiana, condujo al abordaje teórico de los principios motivacionales de autores como Maslow (2006) y especialmente Herzberg (1962), con su teoría de "higiene-motivación" desarrollada a finales de la década de 1950. De acuerdo con este último, el nivel de rendimiento de las personas variaba en función del nivel de satisfacción; es decir, las respuestas hacia el trabajo eran diferentes cuando el individuo o el grupo se sentían bien o cuando se sentían mal. Para él existían cinco factores en la satisfacción laboral: responsabilidad, promoción, realización, reconocimiento y trabajo en sí mismo.

Estos cinco elementos debían verse reflejados en la capacidad de promover el trabajo en grupo. En ese sentido, el ingeniero industrial, entonces estudiante del programa de doctorado de Comportamiento Organizacional de la universidad de Northwestern, en Illinois, y docente de la Universidad de los Andes, Enrique Oligastri (mar.-abr. 1971) publicó un artículo a comienzos del año 1971 donde señalaba la importancia del establecimiento del método vivencial en la dinámica de grupos. Referenciando principalmente los planteamientos teóricos del psicólogo norteamericano Carl Rogers (1961), ${ }^{1}$ Oligastri afirmó que dicho método era posible de aplicar para el desarrollo de los grupos de trabajo y enfoques administrativos. Así, los individuos debían auto-examinarse, procurando experimentar con formas alternativas de relacionarse o comportarse en los lugares de trabajo. De acuerdo con él, los individuos abiertos a los cambios, interesados en aprender permanentemente, capaces de establecer relaciones interpersonales, tolerantes y con una salud mental "normal" (sin definir a qué se refería con normal), eran quienes más provecho podían sacar de este método vivencial (Oligastri, mar.-abr. 1971, p.64).

\section{Cuantificación de lo motivacional}

A partir de lo anterior, se puede decir que, hasta ahí, el tema de la motivación aún se regía bajo la premisa de la racionalización del trabajo para aumentar la eficiencia. Sin embargo, la década de 1970 marcó un punto de quiebre en el abordaje sobre dicho asunto. Durante comienzos de ese periodo, no solo se observó un impulso al modelo de desarrollo basado en la industrialización y crecimiento urbano (Castaño, 2018), sino que también se experimentó la diversificación de las profesiones universitarias y los empleos de oficina (López, 2003). La complejidad y heterogeneidad de esta estructura laboral, ligada con las 
empresas de servicios, coincidió con el perfeccionamiento de una serie de dispositivos destinados a cuantificar los rasgos motivacionales en individuos y grupos de trabajo, con el objetivo de maximizar las estrategias de producción y gestión emocional. Así, en primer lugar, el examen más detallado de los sentimientos subjetivos y grupales adquirió un mayor protagonismo como esfera capaz de evaluarse desde el trabajo de campo. En segundo lugar, la medición y el diseño de cuestionarios y estadísticas también marcaron una huella importante a la hora de cuantificar los niveles de motivación y satisfacción. En 1972 Beltrán (1972) aplicó a sesenta ejecutivos de gerencia y administración la escala de actitudes hacia el trabajo de Frederick Herzberg. Un año después se realizó un estudio en Bavaria que procuró definir los niveles de satisfacción en sus empleados, pero desde una perspectiva asociada con los planteamientos del mismo Herzberg. En seguida fue John Sudarsky (1974), un ingeniero industrial con master en psicología de la Universidad de Kansas, quién llevó a cabo un estudio más detallado que Beltrán para medir el perfil motivacional colombiano, tomando como base una muestra de 710 personas estratificadas según clase, edad, sexo y educación.

Este proceso de medición de la motivación debía partir, en primer lugar, de la elaboración de una prueba para calcular la preponderancia motivacional de los individuos. En segundo lugar, se debía realizar un modelo que permitiese interpretar los resultados. En tercer lugar, se diseñó una muestra representativa que incluyese las diferentes regiones del país, partiendo de la clasificación elaborada por la antropóloga Virginia Gutiérrez de Pineda (1975): zona andina, santandereana, antioqueña, zona de transición, ubicada entre Antioquia y el litoral fluvio minero; en cuarto lugar la elaboración de hipótesis. Sudarsky (1974, p.97) concluía que el ciclo de las desigualdades económicas y sociales tenían como sustrato un fenómeno de diferencias motivacionales, lo que implicaba la psicologización de una serie de problemas de corte social. Para él era esencial modificar la estructura motivacional de determinados grupos, además de identificar aquellos que presuntamente impedían el progreso y explicaban la creciente marginación de amplios sectores poblacionales. Dentro de este análisis era inexistente cualquier tipo de mención a los contextos socioeconómicos como causales, no solo de la referida desigualdad, sino también de la conflictividad reinante en las relaciones laborales a nivel nacional. La identificación de aquellos grupos e individuos que impedían el mencionado "progreso" reflejaba la existencia de un conflicto de intereses que se tornaba necesario neutralizar y reencauzar desde la implementación de este mecanismo psicológico-administrativo.

Estos planteamientos se desarrollaron justo en un periodo histórico del Frente Nacional en donde se constató una fuerte conflictividad entre los trabajadores (organizados bajo parámetros cada vez más contestatarios) y una parte del establecimiento político y económico (Castaño, 2015, p.38). Lo anterior se evidenció a través de cuatro fenómenos. En primer lugar, por medio de una amplia campaña anticomunista destinada a mitigar los conflicto de clases. En segundo lugar, con la restricción del derecho de huelga, tal como sucedió con el decreto legislativo n.939 de 1966 (Colombia, 20 abr. 1966). En tercer lugar, y en contraste con aquella política de restricción, se constató una elevada tasa de sindicalización, que, por ejemplo, se duplicó entre 1959 y 1965 (Hernández, 2014, p.215), y en 87\% entre las décadas de 1960 y 1970 (Delgado, nov. 1998). En cuarto lugar, se evidenció 
una ampliación del proceso organizativo en otros sectores ligados con la banca, la salud y el transporte (Sánchez, 2009, p.133). Estas expresiones reivindicativas permiten considerar que la implementación de aquella estrategia de medición de la motivación respondió a un entorno poco armónico, beligerante, por lo cual era necesario brindar una explicación científica y satisfactoria frente a aquel entorno polarizado. Se anhelaba que dicha explicación sobre un panorama convulso en lo social y laboral contribuyera a sentar las bases para la implementación de un dispositivo psicológico, capaz de identificar, intervenir y desactivar los potenciales conflictos de clase.

Por otro lado, y de acuerdo con los resultados, aquella estrategia también buscaba predecir cuál sería la dinámica social, resultado de las motivaciones personales y de grupos (Sudarsky, 1975, p.97). El modelo de condicionamiento motivacional aplicado por Sudarsky partía de un principio semejante al de la socialización desarrollada por Durkheim (1985), según el cual los diferentes sistemas económicos y sociales que suscitaban el desarrollo del individuo lo iban condicionando paulatinamente en su progreso ulterior. Por consiguiente, la estructura social condicionaba la estructura familiar dentro de la cual el niño era educado. Una vez fuese procesado el individuo, a través del sistema educativo, era incorporado a la fuerza de trabajo.

El resultado de ello debía traducirse, por un lado, en el diseño de un cuestionario estructurado que comparara cuantitativamente los factores obtenidos por el análisis, y, por el otro, con las variables de satisfacción. De igual forma, a partir de la primera mitad de la década de 1980, la incorporación de valores cuantitativos para calibrar los niveles de satisfacción o insatisfacción también posibilitó el desarrollo de ciertas características motivacionales de grupos ubicados en varias categorías ocupacionales. Toro Álvarez y Cabrera Gómez (1985) realizaron un análisis con base en datos obtenidos mediante la aplicación de cuestionarios motivacionales para diferentes grupos ocupacionales a nivel nacional. En ellos se encontraron referencias directas de los psicólogos Hackman y Lawler (1971), quienes, a comienzos de 1971, es decir, casi una década atrás, desarrollaron cuatro condiciones motivacionales básicas de un puesto de trabajo: variedad, autonomía, retroalimentación e identidad de la tarea. Tomando en consideración lo anterior, Toro Álvarez y Cabrera Gómez desarrollaron una serie de caracterizaciones motivacionales para diferentes grupos profesionales, vinculados con varias entidades: el Instituto Agropecuario (ICA), Instituto de Seguros Sociales, Interconexión Eléctrica S.A. (ISA), Instituto Nacional de Educación Media Diversificada (Inem de Medellín), Policía Nacional y Universidad de Eafit. También se hizo el perfil motivacional de bachilleres agropecuarios de varias regiones del país, contadores públicos, ingenieros, médicos y odontólogos, secretarias, agentes de policía, empleados administrativos, entre otros (Toro Álvarez, Cabrera Gómez, 1985, p.192).

\section{El empuje de la voluntad y el clima organizacional}

A juzgar por las fuentes disponibles (Sudarsky, 1974, p.4), a mediados de la década del 1970, caracterizada por el agotamiento del Frente Nacional, el acelerado proceso de urbanización (Palacios, 2008, p.128) y el incesante flujo poblacional del campo a las ciudades en busca de nuevas fuentes de empleo (Gobierno..., 22 ago. 1997), se fue articulando la motivación 
como campo de estudio de las relaciones humanas y desde una base experimental, con el concepto de "clima organizacional". Dicho concepto fue señalado por primera vez, a finales de la década de 1930, por el psicólogo social alemán Kurt Lewin, para quien la interacción entre el ambiente y los individuos definía las formas de comportamiento y el "clima social" (Lewin, Lippitt, White, mayo 1939). Partiendo de lo esbozado por este último, Sudarsky $(1975$, p.7) afirmó que dicho clima organizacional era definido, en primer lugar, como el reflejo de una situación grupal de motivación que obedecía a diferentes patrones de comportamiento y como consecuencia de las necesidades sociales. En segundo lugar, lo concebía como parte de las percepciones que el individuo tenía de la organización para la cual trabajaba ${ }^{2}$ y como forma de establecer un nexo entre los objetivos organizacionales con los individuales. Estimular y suscitar en los individuos la energía, la excelencia, el compromiso, el optimismo y la motivación desde el imperativo del trabajo continuo, se fue vinculando con la necesidad, promovida por centros académicos como la ya mencionada Escuela de Administración Pública y Universidad de los Andes, de fomentar la cultura de los objetivos y la competitividad empresarial en las grandes compañías colombianas. Es interesante notar cómo la emergencia del concepto del clima organizacional coincidió con una lenta transformación del aparato productivo a nivel nacional, caracterizado por un incipiente síntoma de des-industrialización (Clavijo, Vera, Fandiño, 2012) y por el fortalecimiento del mercado laboral en sectores como las comunicaciones, el transporte, las finanzas, servicios a las empresas y al gobierno (Bonet, 2006, p.5). Dicho de otro modo, se experimentó un cambio en las características del empleo urbano, lo cual se vio reflejado en nuevas formas de gestionar la subjetividad de los trabajadores. Se buscó incorporar una norma de comportamiento que estimulara la voluntad, la tenacidad en los trabajos desempeñados hasta el límite de lo posible.

En el proceso de medición del clima organizacional elaborado en la Universidad de los Andes por Alberto Bravo, Víctor Manuel Hoyos y el propio Sudarsky (1974), se tuvo como referencia directa el estudio realizado en 1968 por los psicólogos Litwin y Stringer (1968) en la General Electric, el cual poseía varias dimensiones dentro del proceso de cuantificación: conformidad, responsabilidad, normas de excelencia, recompensas, claridad organizacional, apoyo, seguridad y salario. También se definieron varias etapas como parte de la prueba, las cuales fueron aplicadas a una organización financiera estatal, una compañía manufacturera de bebidas, una empresa de cueros y una organización bancaria privada, ubicadas todas en la ciudad de Bogotá (Sudarsky, 1975, p.12). La muestra fue seleccionada de modo que cubriera una línea jerárquica desde la cabeza de la organización hasta la base, para un total de 234 personas encuestadas: presidente (1), alta gerencia (24), equipos de apoyo a la alta gerencia (31), mandos medios (41), personal de oficina (73), supervisores (17), obreros (47) (Sudarsky, 1974, p.24). La primera de dichas etapas consistía en determinar los grupos o los individuos a quienes se aplicaría aquella prueba, definida por él como la prueba Tecla, además de definir las áreas relevantes con base al conocimiento de la organización. La segunda era precisar las variables del análisis (sexo, educación, antigüedad, salario, unidad de trabajo, nivel jerárquico, región donde trabajaba) e identificar las necesidades como parte de un primer ejercicio exploratorio. La tercera etapa era el diseño que, a su vez, estaba subdividido en otra serie de procesos: desarrollo de un marco teórico y de la 
hipótesis, adaptación del instrumento de medición y procesamiento de la información. La cuarta etapa consistía en la implementación y administración directa del cuestionario. La quinta era el procesamiento de los datos. La sexta era el análisis de los datos para determinar qué aspectos del clima organizacional estaban bajos, como por ejemplo los propósitos de excelencia. La octava era el plan de acción, consistente en el diseño de una estrategia de desarrollo organizacional en el que se determinarían los objetivos de cambio y, de acuerdo con el diagnóstico, se realizarían las intervenciones posibles. La novena era la efectiva implementación y la décima era la medición subsecuente del clima organizacional (Sudarsky, 1974, p.27).

Detrás de ello existía un propósito por implementar estrategias de sondeo, un proceso de cuantificación y análisis de datos. Todo ello a partir de un ejercicio diagnóstico para detectar problemas de motivación y de una intervención sobre la psique para obtener un cambio en las actitudes, valiéndose de ejercicios "correctivos". Una vez obtenido el diagnóstico basado en el procesamiento y gratificación de los resultados se realizó el proceso de retroalimentación con los empleados, además de la ejecución de un plan de reestructuración de las empresas. Cada una de estas etapas desagregadas y sistematizadas reflejaba la intención de hacer de los lugares de trabajo una especie de laboratorio social que operara en función de unos objetivos específicos, unos criterios de observación, clasificación, experimentación, diseño y optimización. Se pretendía que los datos arrojados se pudiesen identificar, computarizar, además de evaluar los resultados de las intervenciones y hacer las correcciones pertinentes desde el plano psicológico-administrativo. Dicha disposición para medir el clima organizacional y pregonar la capacidad de adaptación se fue consolidando durante la década de 1980, justamente cuando se hizo más evidente la disminución del crecimiento económico y la contracción en la generación de empleos. Así, el crecimiento económico del PIB en la década de 1960 había sido del 5.1\% anual promedio, en la década de 1970 fue del 5.5\%, mientras que en la siguiente fue de tan solo 3.2\%. A su vez, la tasa de desempleo aumentó del 8.1\% en 1981 a 9.57\% en 1989, con una cifra record de 13.8\% en 1985, a la vez que se disparó el empleo informal (Perry, 7 ago. 1990).

Dicho de otro modo, la contracción del gasto social por habitante y el aumento en las cifras de desempleo (Perry, 7 ago. 1990) coincidieron con la puesta en marcha de un proyecto de reingeniería organizacional. A modo de ilustración, a finales de 1987, Octavio García, docente de la Facultad de Administración de la Universidad del Valle, desarrolló un modelo de autodiagnóstico de clima organizacional. Para ello diseñó un instrumento guía con el fin de llevar a cabo una serie de entrevistas personales con preguntas como la siguiente: ¿Qué opinión tiene de la empresa? ¿Conoce usted los objetivos generales de la empresa? ¿Cómo percibe usted el ambiente general? ¿Siente usted presión en su trabajo? ¿A través del trabajo usted desarrolla los objetivos personales? ¿Cómo se siente en la empresa? (Perry, 7 ago. 1990, p.75). En general, las preguntas giraban en torno a las siguientes variables: características de la gente, conocimiento de los objetivos, participación en la fijación de los objetivos, compromiso para el logro de los objetivos, percepción del ambiente, presión en el trabajo, evaluación sistemática, logro de objetivos personales, motivaciones percibidas, entre otras.

Hasta ahí el análisis de las motivaciones giró en torno a los incipientes desarrollos teóricos de autores como López y Madariaga durante la primera mitad del siglo. Luego 
se hizo énfasis en sus bondades a través de dispositivos de medición y de intervención desde las décadas de 1960 y 1970 en adelante. Sin embargo, en 1987 los planteamientos de Alonso Arenal consistieron en una previa definición y clasificación de las motivaciones, no solo como condición para una intervención más exitosa y detallada, sino también como una forma de objetivar el recurso humano desde una perspectiva psicosocial, cultural y organizacional. En resumen, para él existían las motivaciones objetivas y subjetivas. Las primeras eran impulsadas por acciones exteriores al individuo, mientras que las segundas valoraban sus fuerzas interiores. La concepción objetivista distinguía tres clases de motivaciones: la motivación-necesidad, la cual consistía en el conjunto de condiciones ambientales que imponían vías obligadas al comportamiento del individuo a nivel biológico, cultural y organizacional. Las motivaciones-aspiraciones, las cuales se traducían en tensiones y objetivos inducidos por fenómenos colectivos como las normas y estereotipos sociales. Las motivaciones-artificiales, denominadas también como incentivos o estímulos institucionalizados por el medio exterior. La concepción subjetivista se centraba en los factores internos y en los deseos, constituidos en la fuente principal de las iniciativas individuales (Perry, 7 ago. 1990, p.10).

\section{Calidad de vida como fuente de rendimiento laboral}

La articulación de la satisfacción, clima laboral, prácticas evaluativas y rendimiento se llevó a cabo en medio de una incertidumbre laboral que fue vista, desde esta óptica, como promotora de acciones eficientes y rápidas. La comodidad otorgada por la estabilidad laboral se representó como un aspecto desencadenante de prácticas improductivas, "paquidérmicas" y presuntamente "conformistas" (Calle Correa, Vieira Mejía, Marín Álvarez, 1991, p.61). La relación entre tensión mental y adaptabilidad, sumada a la demanda de mayor esfuerzo, movilización en tiempo (Lipovetsky, 1994, p.276) y sentimiento de urgencia, tuvo como correlato la necesidad de enarbolar el concepto de calidad de vida en el trabajo. Se trató de una estrategia destinada a atenuar los efectos psicológicos de la crisis en el empleo experimentada en ese periodo y acentuada con la expedición de la ley n.50 de 1990. Esta normatividad tuvo como consecuencia la liberación de las presuntas rigideces del sistema laboral a través de la legalización de los empleos temporales, contratos a término fijo y diversas modalidades de subcontratación, con el fin de mejorar las también supuestas condiciones de "competitividad" de las empresas colombianas (Bogotá, 28 dic. 1990).

En medio de aquel contexto caracterizado por la apertura económica y la desregulación del mercado laboral, autores como Toro Álvarez (1991, p.33) definieron la calidad de vida en el trabajo a partir de la percepción subjetiva del individuo, partiendo de variables como la experiencia en el trabajo y el nivel de educación. Es interesante notar cómo, a medida que se operaba un tránsito hacia una modelo de apertura económica y las condiciones del mercado laboral se tornaban más difíciles, también se fue recurriendo a un discurso psicológico orientado a paliar sus efectos y a hacer más soportables aquellas condiciones externas. De modo que al señalar los análisis desarrollados por los psicólogos organizacionales norteamericanos Philip H. Mirvis y Edward E. Lawler (1984), Toro planteaba que los criterios de calidad de vida en el trabajo eran diversos debido a la concepción que de ella tenían 
diferentes disciplinas, y a las nociones cambiantes de empresas y empleados. Más allá de ello, existían dos aspectos comunes a una definición de calidad de vida en el trabajo: la primera, relacionada con las características y el ambiente de trabajo que afectaba la vida laboral de los empleados; y, la segunda, relacionada con los criterios de seguridad y bienestar. En general, ambos autores norteamericanos, retomados por Toro, definían la calidad de vida en el trabajo como aquella que promovía la vinculación de los empleados, los entrenaba, los desarrollaba, les proporcionaba experiencias enriquecedoras, los estimulaba a participar en decisiones relativas con el puesto que ocupaba, además de proveerles un trato justo (Mirvis, Lawler, 1984, p.20). Tomar en consideración estos elementos contribuía, según Toro, a evitar las enfermedades mentales, el alcoholismo, la corta esperanza de vida y la poca participación en la vida comunitaria (p.39). A pesar de que para él no existía mayor compromiso por parte de las empresas colombianas a la hora de asumir esta "causa" (p.39), era necesario preconizar sus beneficios para potenciar los rendimientos, capacidades, talentos, aptitudes y bienestar del individuo.

Si para Sudarsky $(1975$, p.7) el clima organizacional se enfocaba en el tema de las motivaciones y percepciones que el individuo tenía de la organización para la cual trabajaba, la calidad de vida se refería a una especie de relación económica, social y psicológica entre la organización, la comunidad y los empleados. De igual manera, Toro Álvarez (1991, p.33) se valió de lo planteado por los psicólogos norteamericanos Davis y Cherns (1975) para afirmar que, a diferencia de aquellas categorías calculables, medibles y objetivables desde un lenguaje estadístico y matemático, como motivación, satisfacción y clima organizacional, la calidad de vida en el trabajo permitía observar fenómenos mucho más generales, dinámicos y que tomaran en consideración las necesidades conjuntas del trabajador, el empleador y la comunidad, vistos como potenciales consumidores. Se trataba de concebir las organizaciones como estructuras mucho más complejas que simples entidades biológicas sin pasado, sin futuro, sin capacidad de modificarse y, por tanto, fácilmente medibles y cuantificables (Toro Álvarez, 1991, p.35). Del mismo modo que el trabajador necesitaba ser creativo, participativo y, por lo tanto, productivo y eficiente, aquellos requerimientos debían asentarse, presuntamente, en un trasfondo psicológico de bienestar y, sobre todo, anhelo de felicidad (p.35) en el plano laboral y doméstico. Así, finalmente, la felicidad se fue articulando al proyecto de maximizar los beneficios empresariales. La justificación de la felicidad en un periodo marcado por la progresiva apertura de los mercados y por el incremento del desempleo, sumado al proceso de desindustrialización (López Gómez, 14 oct. 1997) experimentado ya desde la segunda mitad de la década de 1970, también tuvo su reverso en la supuesta desmotivación ocasionada por el fenómeno del desempleo y la inestabilidad laboral que aquejaba a un creciente contingente de colombianos (García, 1987, p.24).

\section{Consideraciones finales}

Es interesante resaltar que a partir de la década de 1960 aspectos como la satisfacción y la motivación fueron cobrando mayor fuerza en el ámbito laboral, a medida que el país transitaba por una etapa de sustitución de importaciones. Este último fenómeno 
fortaleció el proceso de industrialización y diversificación del aparato productivo nacional, lo cual tuvo una estrecha relación con la emergencia y consolidación de los dispositivos psicológicos a partir de la segunda pos guerra. El individuo objetivado en estas instancias comenzó a ser percibido como un actor social cuya conducta, sentimientos, percepciones, emociones, estarían sometidos a una racionalidad estratégica. Este enfoque iniciado por la caracterización del factor humano en términos de "recurso" (Galvis González, 2010, p.74), fue haciendo mayor énfasis en los temas de la iniciativa, el compromiso y la motivación (Dejours, 1998, p.23). Si bien en autores como López y Madariaga aspectos como la motivación estuvieron presentes en sus planteamientos, durante la segunda mitad del siglo XX fue mucho más evidente y detallada esta concepción del individuo. Lo anterior partía de la idea de verlo como un actor sobre el cual debía de suscitarse un conjunto de conductas motivacionales, tomando en consideración dos aspectos. Por un lado, las características emotivas y personales que debía poseer el líder empresarial; y, por otro lado, las condiciones emocionales que debían promoverse en el obrero y empleado para afianzar el proceso de fortalecimiento del aparato productivo nacional.

También se constató que, a partir de mediados de la década de 1970, se fue promoviendo, en primer lugar, una nueva articulación entre la motivación como campo de estudio de las relaciones humanas, desde una base experimental y cuantificable, con el concepto de "clima organizacional". El lenguaje matemático y el trabajo de campo se constituyeron en una serie de fórmulas más precisas a la hora de procurar gestionar y reconducir el personal en el ámbito laboral. A partir de esa época, justo cuando comenzó a experimentarse un creciente proceso de desindustrialización, fortalecimiento de la economía de servicios y posterior contracción del empleo, dicha gestión empezó a descansar en el imperativo de la excelencia, el compromiso y la competencia en las empresas colombianas, con el propósito de extraer al máximo las capacidades individuales y colectivas. Invocar la voluntad y la disposición emocional bajo los imperativos de la excelencia y la competencia, se erigió en la herramienta idónea para hacerle frente a un imperativo social caracterizado por la supervivencia del más apto en términos económicos y laborales.

Lo anterior también respondió a un mayor nivel de circulación, filiación y apropiación conceptual de la psicología organizacional norteamericana en el ámbito de la producción nacional, especialmente en centros educativos como la Universidad de los Andes. En segundo lugar, a partir de la década de 1990, en un momento de crisis económica, aumento en la tasa de desempleo, emergencia de nuevas y precarias formas de vinculación laboral, sumado a la internacionalización de la economía durante la presidencia de Cesar Gaviria, la búsqueda de la felicidad se constituyó en una estrategia para gestionar el clima organizacional y mejorar las posibilidades de producción de los trabajadores en las empresas. El aforismo de la felicidad no sólo se erigió en un estado emocional deseable para la plenitud individual y colectiva, sino que también se fue estableciendo en una especie de fórmula destinada a garantizar la estabilidad emocional, productividad y cohesión en los lugares de trabajo. Esta perspectiva orientada a incorporar estándares de calidad de vida, buen clima organizacional y felicidad en el plano laboral entrañó una especie de misión administrativa destinada a promover actitudes productivas. Se fue llevando a cabo un escrutinio mucho más detallado sobre un conjunto de experiencias, sentimientos y sensaciones ligados con 
la vida intima. Detrás de ello se advirtió la finalidad de neutralizar los conflictos de clase, optimizar rendimientos e instalar capacidades de adaptación en un mundo del trabajo donde el nivel de competitividad tendió a incrementarse y la inestabilidad laboral se fue haciendo más notoria.

\section{AGRADECIMIENTOS}

Este artículo es el resultado de una tesis doctoral en historia, Cuerpos y almas para el trabajo: la psicologización de los trabajadores en Bogotá y Medellín, 1928-1994, que fue desarrollada en la Universidad Nacional de Colombia (UNC), sede Medellín. El director de la tesis fue Álvaro Andrés Villegas Vélez; el co-director, Óscar Fernando Gallo Vélez. Media beca brindada por la UNC, sede Medellín.

\section{NOTAS}

${ }^{1}$ El grupo vivencial fue una concepción de trabajo con grupos, desarrollada por la psicología o psicoterapia fenomenológica existencial durante la década de 1960. Carl Rogers procuró incorporar un enfoque bajo el cual se debía procurar exaltar la autonomía, emotividad e individualidad de las personas participantes en los procesos terapéuticos y en las interacciones grupales. Oligastri (mar.-abr. 1971) procuró adoptar parte de este enfoque psicológico dentro de sus teorías administrativas. Ver Rogers (1961).

${ }^{2}$ Esta definición que alude al clima organizacional como un asunto ligado con las percepciones fue tomado, a su vez, de los desarrollos teóricos del Francis Cornell. Para él, el clima organizacional partía de una mezcla de percepciones que los individuos desarrollaban en una organización con relación a los compañeros, de modo que las percepciones definían dicho clima. Consultar Cornell (1955).

\section{REFERENCIAS}

AQUILINO, Natalia.

Cuerpo y salud mental en la problemática del trabajo. Estudio (Maestría em Ciencias Sociales del Trabajo) - Universidad de Ciencias Empresariales y Sociales, Buenos Aires. 2004.

BELTRÁN, Andrés.

Comparación de la teoría de F. Herzeberg sobre motivación, higiene y análisis de acuerdo a ella de una empresa colombiana. Bogotá: Publicaciones de la Facultad de Ingeniería/Universidad de los Andes. 1972.

BILLIARD, Isabelle.

Les pères fondateurs de la psychopathologie du travail en butte a l'enigme du travail. Cliniques Méditerranéenes. 2002.

BOGOTÁ.

Ley n.50, de 28 diciembre 1990, por el cual se introducen reformas al Código Sustantivo del Trabajo y se dictan otras disposiciones. Disponible en: <http://www.alcaldiabogota.gov. co/sisjur/normas/Norma1.jsp?i=281>. Acceso en: 28 dic. 1990.

BONET, Jaime.

La tercerización de las estructuras económicas regionales en Colombia. Cartagena: Banco de la República. (Documentos de trabajo sobre economía regional, n.67). Disponible en: <http://www.banrep.gov.co/sites/default/files/ publicaciones/archivos/DTSER-68_\%28VE\%29. pdf $>$. Acceso en: 9 abr. 2020. ene. 2006.

CALLE CORREA, Olga Lucía; VIEIRA MEJÍA, María del Pilar; MARÍN ÁLVAREZ, Mónica María.

Estudio descriptivo de la satisfacción y el clima organizacional en relación con el rendimiento laboral en una empresa privada del Valle de Aburrá, Medellín. Tesis (Pregrado en Psicología) Universidad de San Buenaventura, Bogotá. 1991.

CAPONI, Sandra.

Del culto a la laboriosidad a la preocupación por la fatiga: dos miradas sobre la pobreza (Argentina, 1903-1904). Mundos do Trabalho, v.7, n.13, p.43-64. 2015.

CASTAÑO, Eugenio.

Cuerpo y alma en las políticas de bienestar:

Medellín, 1945-1975. Salamanca: Levar Anclas

Ediciones. 2018.

CASTAÑO, Eugenio.

Fisonomías del miedo: un paulatino enmudecimiento. Recuento de luchas y lógicas de la violencia antisindical en el departamento del Atlántico: CUT, Sintraelecol y Anthoc, 1975 2012. Medellín: Editorial Escuela Nacional Sindical. 2015. 
CLAVIJO, Sergio; VERA, Alejandro; FANDIÑO, Alejandro.

La desindustrialización en Colombia: análisis cuantitativo de sus determinantes. Bogotá: Centro de Estudios Económicos Anif. Disponible en: <http://anif.co/sites/default/files/uploads/ Anif-Desindustrializacion-12.pdf $>$. Acceso en: 9 abr. 2020. 2012.

\section{COLOMBIA.}

Decreto n.939 de 20 de abril de 1966, por el cual se dictan disposiciones de carácter laboral. Disponible en: <http://www.suin-juriscol.gov. co/viewDocument.asp?ruta=Decretos $/ 1193776>$. Acceso en: 19 mar. 2020. 20 abr. 1966.

CORNELL, Francis.

Socially perceptive administration. New York: Ronald Press. 1955.

DÁVILA, Carlos.

Teorías organizacionales y de administración: enfoque crítico. Bogotá: McGraw Hill. 2001.

DAVIS, Louis E., CHERNS, Albert (Ed.). The quality of working life. New York: Free Press, McMillan. 1975.

DEJOURS, Christophe.

El factor humano. Buenos Aires: Lumen. 1998.

DELGADO, Álvaro.

Las luchas laborales en Colombia: coyunturas y perspectiva inmediata. Credencial Historia, n.107. nov. 1998.

DURKHEIM, Émile.

La división del trabajo social. v.1-2. Barcelona: Planeta Agostini. 1985.

FRIEDMAN, Georges.

Sociología del trabajo. Ciudad de México: Fondo de Cultura Económica. 1971.

GALLO VÉLEZ, Óscar.

Luz sobre el fenómeno de un corazón aparentemente infatigable: la fatiga de la clase obrera en Colombia, 1898-1946. In: Gallo Vélez, Óscar; Castaño, Eugenio (Ed.). Salud laboral en el siglo XX y XXI: de la negación al derecho a la salud y la enfermedad. Medellín: Ediciones Escuela Nacional Sindical. p.219-252. 2016a.

GALLO VÉLEZ, Óscar.

Trabalho, medicina e legislação na Colômbia, 1910-1946. Tese (Doutorado em História) - Universidade Federal de Santa Catarina, Florianópolis. 2016b.

GALVIS GONZÁLEZ, José Ariel.

Factor humano en el trabajo desde la perspectiva de escuela de las relaciones humanas: reflexión para la región. Páginas: Revista Académica e Institucional de la UCPR, n.86, p.67-78. 2010.
GARAY, Jorge Luis.

Colombia: estructura industrial e

internacionalización, 1967-1996. Santa Fe de Bogotá: Departamento Nacional de Planeación. 1998.

GARCÍA, Octavio.

Un modelo para el autodiagnósitco del clima organizacional. Revista Tecnología Administrativa, v.5, n.12, p.68-88. 1987.

GOBIERNO....

Gobierno de Misael Pastrana Borrero. El Tiempo, 22 ago. 1997.

GUTIÉRREZ DE PINEDA, Virginia.

Familia y cultura en Colombia: tipologías, funciones y dinámica de la familia: manifestaciones múltiples a través del mosaico cultural y estructuras sociales. Medellín: Editorial Universidad de Antioquia. 1975.

HACKMAN, Richard; LAWLER, Edward. Employee reactions to job characteristics. Journal of Applied Psychology, v.3, n.55. p.182-186. 1971.

HAIDAR, Victoria.

'Buscad al hombre': la problematización del factor humano en la explicación de los accidentes de trabajo: Argentina, entre la actualidad y la historia. In: Gallo Vélez, Óscar; Castaño, Eugenio (Ed.). Salud laboral en el siglo $X X$ y XXI: de la negación al derecho a la salud y la enfermedad. Medellín: Ediciones Escuela Nacional Sindical. p.253-284. 2016.

HERNÁNDEZ, Francisco.

El sindicalismo en Colombia: implicaciones sociales y políticas. Trabajo de grado (Grado en Derecho) - Pontificia Universidad Javeriana, Bogotá. 2014.

HERZBERG, Frederick.

The motivation to work. New York: John Wiley and Sons. 1962.

HOYOS, Víctor Manuel; BRAVO, Alberto; SUDARSKY, John.

Diseño de un instrumento para medir clima organizacional. Bogotá: Universidad de los Andes. 1974.

ILLUZ, Eva.

Intimidades congeladas: las emociones en el capitalismo. Madrid: Katz. 2006.

JARABA BARRIOS, Bruno Andrés.

Un escritorio para Mercedes: revisando el mito fundacional de la psicología en Colombia. Tesis (Maestría en Sociología) - Universidad Nacional de Colombia, Bogotá. 2014.

JARAMILLO, Germán.

Adaptación de la empresa al hombre. Fabricato al Día, v.59, n.5, p.29. 1964. 
LAVAL, Christian; DARDOT, Pierre.

La nueva razón del mundo. Barcelona: Gedisa. 2013.

LE BIANIC, Thomas.

Les conservatoires des arts et métiers et la 'machine humaine': naissance et développement des sciences de l'homme au travail au CNAM, 1910-1990. Revue d'Historie des Sciences Humaines, v.2, n.11, p.185-214. 2004.

LEWIN, Kurt; LIPPITT, Ronald; WHITE, Ralph K.

Patterns of aggressive behavior in experimentally created 'social climates'. The Journal of Social Psychology, v.10, p.271-300. mayo 1939.

LIPOVETSKY, Gilles.

El crepúsculo del deber: la ética indolora de los nuevos tiempos democráticos. Barcelona: Anagrama. 1994.

LITWIN, George; STRINGER, Robert.

Motivation and organizational climate. Boston: Graduate School of Bussines Administration/ Harvard University. 1968.

LÓPEZ, Abel Ricardo.

Empleados, mujeres de oficina y la construcción de las identidades de clase media en Bogotá, 1930-1950. Anuario Colombiano de Historia Social y de la Cultura, n.30, p.257-279. 2003.

LÓPEZ, Alejandro.

El trabajo: nociones fundamentales. Medellín: Fondo Editorial Universidad de Eafit. 2011.

LÓPEZ GÓMEZ, Edmundo.

Apertura y desempleo. El Tiempo, 14 oct. 1997.

LOTERO ZAPATA, Raúl.

Producir bien. Fabricato al Día, v.59, n.5, p.19. 1964.

MADARIAGA, Cesar de.

Introducción al estudio del factor humano en la indústria: nociones de psicoeconomía. Bogotá: Talleres de la Cooperativa Nacional de Artes Gráficas. 1946.

MASLOW, Abraham.

Motivación y personalidad. Madrid: Díaz de Santos. 2006.

MIRVIS, Philip; LAWLER, Edward.

Accounting for the quality of work life. Journal of Occupational Behavior, v.5, n.3, p.197-212. 1984.

MONTOYA, Hernando.

Salud mental. Fabricato al Día, p.8-9, sep.-oct. 1966.

MOTTA, Julia María.

Fragmentos da história e da memória da psicologia no mundo do trabalho no Brasil: relações entre a industrialização e a psicologia. São Paulo: Ágora. 2008.

OCAMPO, José Antonio.

Historia económica de Colombia. Bogotá: Siglo XXI. 1987.

OLIGASTRI, Enrique.

Notas introductorias al método vivencial en dinámica de grupos. Razón y Fábula, v.24, p.2243. mar.-abr. 1971.

ORIENTACIÓN.

Orientación. Fabricato al Día, v.16, n.2, p.11. 1960.

OUVRIER-BONNAZ, Régis.

L'historie des chaires du CNAM concernant l'homme au travail (1900-1945): entre production de saviors et engagement politique. Les Cahiers d'Historie, v.111, p.99-122. 2010.

PALACIOS, Marco (Comp.).

Historia general de América Latina. v.8. Paris: Ediciones Unesco. 2008.

PERRY, Guillermo.

Una década gris oscura. El Tiempo, 7 ago. 1990.

POHL-VALERO, Stefan.

Termodinámica, pensamiento social y biopolítica en la España de la restauración. Universitas Humanística, n.69, p.36-60. 2010.

PULIDO MARTINEZ, Hernán Camilo.

Work and the constitutions of the subject: the case of the urban passenger transport in the Bogotá, Colombia. Tesis (Doctorado en Psicología) School of Social Sciences, Cardiff University, Cardiff. 2008.

RABINBACH, Anson.

The human motor: energy, fatigue, and the origins of modernity. Los Angeles: University of California Press. 1990.

RAMÍREZ CARDONA, Carlos.

Notas sobre administración científica: la motivación en el trabajo. Bogotá: Imprenta Nacional; Escuela Superior de Administración Pública. 1963.

ROGERS, Carl.

On becoming a person: a therapists view of psychotherapy. San Diego: Houghton Mifflin. 1961.

ROLDAN, Diego.

Discursos alrededor del cuerpo, la máquina, la energía y la fatiga: hibridaciones culturales en la Argentina de fin-de-siecle. História, Ciências, Saúde-Manguinhos, v.17, n.3, p.643-661. 2010.

ROSE, Nikolas.

Governing the soul: the shaping of the private self. London: Routledge. 1990. 
SÁNCHEZ, Ricardo.

Huelga: luchas de la clase trabajadora en

Colombia, 1975-1981. Bogotá: Editorial

Universidad Nacional de Colombia. 2009.

SARRACENO, Marco.

De la mesure du corps à la politique des corps: une historie des sciences du travail, 1880-1920.

Thèse (Doctorat en Sociologie) - Université de

Nanterre, Nanterre. 2013.

SATIZABAL, Ernesto.

La atmósfera psicológica en la empresa. Fabricato al Día, p.26, ago. 1964.

SENNETT, Richard.

La corrosión del carácter: las consecuencias personales del trabajo en el nuevo capitalismo. Barcelona: Anagrama. 2000.

SUDARSKY, John.

Un modelo de diagnóstico e intervención en desarrollo organizacional: la medición del clima organizacional. Bogotá: Universidad de los Andes. 1975.

SUDARSKY, John.

Motivación, individuo y sociedad. Bogotá:

Publicaciones de la Facultad de Ingeniería de la Universidad de los Andes. 1974.

TORO ÁlVAREZ, Fernando.

Calidad de vida en el trabajo, alcances e implicaciones. Revista Interamericana de Psicología Ocupacional, v.10, n.1, p.95-137. 1991.

TORO ÁlVAREZ, Fernando; CABRERA GÓMEZ, Hernán.

Motivación para el trabajo: conceptos, hechos y evidencias contemporáneas. Medellín: Ediciones Gráficas. 1985.

VATIN, François.

Trabajo, ciencias y sociedad: ensayos de sociología y epistemología del trabajo. Buenos Aires:

Lumen-Humanitas. 2004.

\section{$\rightarrow \rightarrow \rightarrow<<<$}




\section{ER R A T A}

En el artículo "Cuerpos y mentes para el trabajo: la psicologización de los trabajadores en Bogotá y Medellín, 1946-1991" (http://dx.doi.org/10.1590/S0104-59702020000200008), publicado en el v.27, n.2, abr.-jun. 2020:

- en la página 447, en el resumen en español, línea 11, donde dice "marca",

- dígase "marcha";

- en la página 447, en el resumen en español, línea 19, donde dice "del",

- dígase "de".

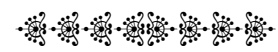

